\title{
A peculiarity of metal-poor stars with planets?
}

\author{
M. Haywood \\ GEPI, Observatoire de Paris, CNRS, Université Paris Diderot, 92190 Meudon, France \\ e-mail: misha.haywood@obspm.fr
}

Received 25 November 2007 / Accepted 12 February 2008

\begin{abstract}
Stars with planets at intermediate metallicities $([-0.7,-0.2]$ dex) exhibit properties that differ from the general field stars. Thirteen stars with planets reported in this metallicity range belong to the thick disc, while only one planet have been detected among stars of the thin disc. Although this statistics is weak, it contradicts the known correlation between the presence of planet and metallicity. We relate this finding to the specific property of the thin disc in this metallicity range, where stars are shown to rotate around the Galaxy faster than the Sun. Their orbital parameters are conveniently explained if they are contaminants coming from the outer Galactic disc, as a result of radial mixing. This must be considered together with the fact that metal-rich stars $([\mathrm{Fe} / \mathrm{H}]>+0.1 \mathrm{dex})$ found in the solar neighbourhood, which are the hosts of most of the detected planets, are suspected of being wanderers from the inner Galactic disc. It is then questionned why stars that originate in the inner and outer thin disc show respectively the highest and lowest rate of detected planets. It is suggested that the presence of giant planets might be primarily a function of a parameter linked to galactocentric radius, but not metallicity. Combined with the existing radial metallicity gradient, then radial mixing explains the correlation at high metallicity observed locally, but also the peculiarity found at low metallicity, which cannot be accounted for by a simple correlation between metallicity and planet probability.
\end{abstract}

Key words. stars: planetary systems - Galaxy: solar neighbourhood

\section{Introduction}

The parent stars of exoplanets are known to be, on the average, more metal-rich than "single" stars of the solar neighbourhood (Gonzales 1997; Santos; Israelian \& Mayor 2000). Apart from this peculiarity, stars with planets show little, if any, specific properties (see Udry \& Santos 2007, for a review). They do not seem to have peculiar chemical abundance ratios (Ecuvillon et al. 2006), although slight differences have been claimed (Robinson et al. 2006), while kinematic behaviour also seems similar to that of field stars (Barbieri \& Gratton 2002). The origin of this preference for metal-rich stars is still discussed, with two possible hypothesis: primordial (Santos et al. 2000) or gained by the star because of a possible infall episode at its birth (Gonzales 1997; Laughlin 2000). Recent advances, observational as well as theoretical (Ida \& Lin 2005; Mordasini et al. 2007) seems to some support the former hypothesis.

Radial mixing, combined with the metallicity gradient in the Galactic disc, is probably responsible for the extended tails of the metallicity distribution of both metal-rich and metal-poor stars of the thin disc in the solar neighbourhood (Haywood 2007). Given that a majority of planets are detected around metal-rich stars, which seem to follow the same kinematic behaviour as the parent metal-rich population, it implies that most would originate from the inner Galactic disc (Ecuvillon et al. 2007). The intermediate metallicity range $([-0.7,-0.2]$ dex $)$ shows a more complex pattern because it involves two distinct populations: the thick disc at $[\mathrm{Fe} / \mathrm{H}]<-0.2$, and the thin disc starting at about $[\mathrm{Fe} / \mathrm{H}]=-0.7$ upward. However, these two populations differ on several points. First, they have distinct kinematic behaviour, the thick disc lagging the Local Standard of Rest (LSR), while the thin disc metal-poor population have $V$ component of space motion distinctly higher than average (see next section). Second, they have different level of $\alpha$ enrichment, with the thick disc at $[\alpha / \mathrm{Fe}]>0.17 \mathrm{dex}$ and the thin disc between 0 . and $0.1 \mathrm{dex}$. Finally, they probably have distinct age distributions. The thick disc or transition stars are essentially old objects (ages $>8$ Gyr), while thin disc metal-poor stars as young as 2 Gyr can be found, even though most are older than this lower limit. These properties, and the hiatus in metallicity between the two populations (the fact that an old population, the thick disc, has stars up to $[\mathrm{Fe} / \mathrm{H}]=-0.2$, while a younger one, the thin disc, has stars down to $[\mathrm{Fe} / \mathrm{H}] \approx-0.7$ ), have been interpreted in Haywood (2007) has testifying the different origins of these two populations: the metal-poor thin disc stars being wanderers from the outer Galactic disc, while the thick disc and transition stars stem from the evolution of the disc at the solar circle.

In the present study, we examine orbital and chemical characteristics of planet host stars to see how they follow this general picture. In the next section, we first show evidences of the radial mixing effects on solar neighbourhood stars, then compare the chemical and kinematics parameters of stars with planets with the general population. We discuss our results in the last section.

\section{A peculiarity of metal-poor stars with planets}

\subsection{Evidences of radial mixing from solar neighbourhood stars}

Thin disc stars at the two extremes of the metallicity interval have markedly different kinematic behaviour. This is easily seen in a plot of the $V$ velocity component (with respect to the Sun) as a function of metallicity (Fig. 1). Figure 1a shows a distribution of these two parameters from various samples with accurate measurements of metallicity : Reddy et al. (2003), Reddy, Lambert \& Allende Prieto (2006), Bensby et al. (2005), 

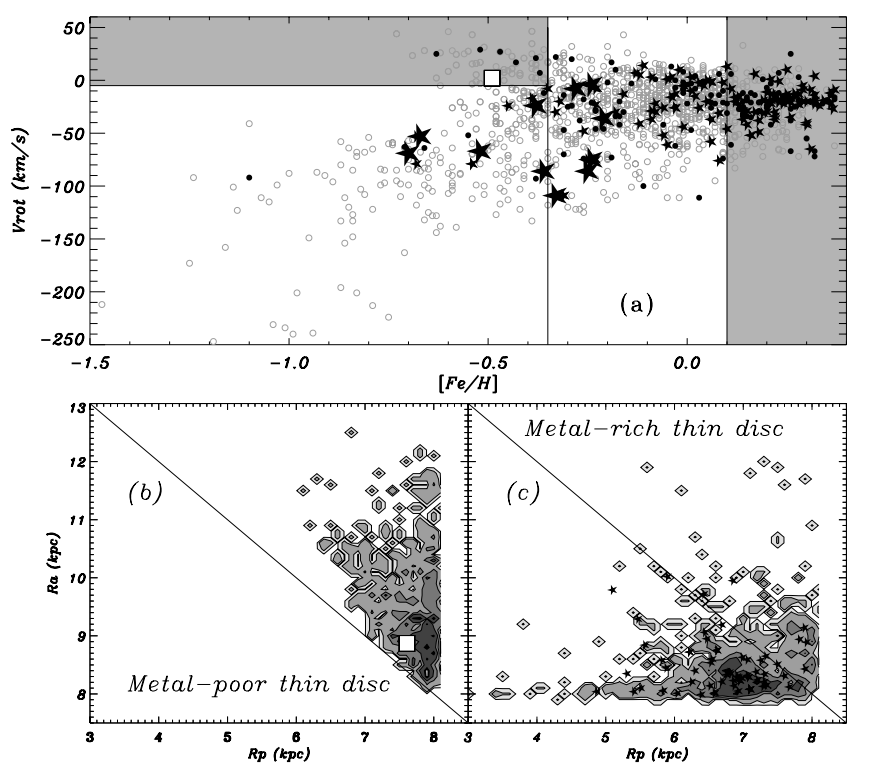

Fig. 1. a) $\left([\mathrm{Fe} / \mathrm{H}], V_{\text {rot }}\right)$ distribution for stars in the samples of Reddy et al. (2003), Reddy et al. (2006), Bensby et al. (2005) and Valenti \& Fischer (2005). Metal-poor stars $(-0.8<[\mathrm{Fe} / \mathrm{H}]<-0.3 \mathrm{dex})$ in this plot separate in two groups: one made of thick disc stars lagging the sun with $V_{\text {rot }}$ within $[-40,-80] \mathrm{km} \mathrm{s}^{-1}$. The other one is preceding the Sun and extending towards $\left([\mathrm{Fe} / \mathrm{H}]=-0.8, V_{\text {rot }}=+60 \mathrm{~km} \mathrm{~s}^{-1}\right)$. Stars with planets from the sample of Gilli et al. (2006) are shown as star symbols, the larger symbols representing the objects of Table 1. Stars with no detected planet from Gilli et al. (2006) are represented by dots. The only star detected with a planet in the metal-poor thin disc group (HD 171028) is represented by a square in panels a) and b). The grey areas give the limits of the two subsamples of thin disc stars with (1) $[\mathrm{Fe} / \mathrm{H}]<-0.35$ and $V_{\text {rot }}>-5 \mathrm{~km} \mathrm{~s}^{-1}$; and (2) stars with $[\mathrm{Fe} / \mathrm{H}]>$ 0.1 dex. Panels b) and c) show the distribution of apo- and peri-centers $\left(R_{\mathrm{a}}, R_{\mathrm{p}}\right)$ for stars in the GCS catalogue within the same limits. Stars with planets and $[\mathrm{Fe} / \mathrm{H}]>0.1$ dex are shown as star symbols in panel c). The line on plot b) and c) materialises an orbit with mean $\left(R_{\mathrm{a}}+R_{\mathrm{p}}\right) / 2=$ $8 \mathrm{kpc}$.

Valenti \& Fischer (2005). These data sets have been crossidentified with the Geneva-Copenhagen Survey (hereafter GCS) (Nordström et al. 2004) in order to obtain the $V$ velocity component of each star. Figure 1a shows that the metal-poor population (which we define here conservatively as $[\mathrm{Fe} / \mathrm{H}]<-0.35 \mathrm{dex}$ ) of the disc separates in two groups. The first one is mostly preceding the LSR, and limited in metallicity to $-0.8<[\mathrm{Fe} / \mathrm{H}]<$ -0.35 dex (grey area in Fig. 1a), with probably few stars only having higher metal content. In the GCS catalogue, stars rotating with the LSR have a mean metallicity of -0.16 dex, while those preceding the LSR by $5 \mathrm{~km} \mathrm{~s}^{-1}$ have a mean metallicity of -0.24 dex, -0.31 dex at $10 \mathrm{~km} \mathrm{~s}^{-1}$, and -0.38 dex at $20 \mathrm{~km} \mathrm{~s}^{-1}$. The second group is lagging the LSR, the lag increasing with decreasing metallicity. These two groups are easily identified as the thin disc and the thick disc, and also correspond to stars with distinctive $\alpha$-element ratios (Sect. 2.2.2).

Because of the position of the Sun at about $8 \mathrm{kpc}$ from the galactic center, its vicinity is likely to be contaminated by both metal-rich stars from the inner disc and metal-poor stars from the outer disc. In order to contrast most clearly the orbital properties of these two groups, we now select them from the GCS catalogue by imposing $V>-5 \mathrm{~km} \mathrm{~s}^{-1}$ and $[\mathrm{Fe} / \mathrm{H}]<-0.35$ dex and metal-rich stars by $[\mathrm{Fe} / \mathrm{H}]>+0.1 \mathrm{dex}$ (grey areas in Fig. 1a). Their apo and peri-centre distributions $\left(R_{\mathrm{a}}, R_{\mathrm{p}}\right)$ are shown on panels (1b) and (1c). The metal-poor group is confined to the upper part of Fig. $1 \mathrm{~b}$ due to the kinematic criterion, while the metal-rich stars occupy an almost distinct area. The group of metal-poor thin disc stars populates mainly outer orbits, or orbits with larger angular momentum, while metal-rich objects symmetrically occupy orbits of lower angular momentum. The fact that low and high metallicity stars are so prevalent among each of these two distinct groups, a property that is expected from the existence of the radial metallicity gradient, clearly indicates that they most probably are the signatures of the radial mixing processes in the Galactic disc (see also Famaey et al. 2007 about the Hyades stream, which conforms well with this general picture). In the scenario presented by Sellwood \& Binney (2002) for example, stars are supposedly loosing or gaining angular momentum at corotation because of spiral waves. This is a secular process, and it takes several gigayears before stars in the outer and inner Galactic disc reach the solar orbit. It means that we may expect to see the signature of this process on stars approaching the solar orbit from the outer or inner disc. In this case they must still appear with orbital characteristics slightly off those of the main population. Because of the radial metallicity gradient, metal-rich stars are expected to come from the inner disc, or to approach the solar circle from smaller radii (Fig. 1c), while metal-poor thin disc stars would come mainly from the outer thin disc (Fig. 1b). If the distribution $\left(R_{\mathrm{p}}, R_{\mathrm{a}}\right)$ of metal-rich stars with planet follows the behaviour of field stars in panel Fig. 1c, it can be deduced similarly that they originate from the inner disc. Stars with $[\mathrm{Fe} / \mathrm{H}]>0.1$ dex from the sample of Gilli et al. (2006) are shown as star symbols in Fig. 1c. They are superimposed with no noticeable differences with field stars. This result must be compared with the distribution of metal-poor thin disc stars of panel (b), where only one star with planet has been found (HD 171028). Since these two groups of stars probably originate from very different galactocentric distances, this may be a hint that a parameter linked to the distance to the galactic center could play a role in the presence of planets. We now discuss if metallicity is the right parameter.

\subsection{The paucity of metal-poor thin disc star with planets}

This work is based on the two samples (stars with and without planets) of Gilli et al. (2006), which is the only study providing measurements of $\alpha$-element abundances for a significant number of stars of both categories. It has been completed for a few lowmetallicity stars with planets from the literature and not available in the selection of Gilli et al. (2006).

\subsubsection{Kinematics}

The sample of stars with known planet (star symbols) and stars with no detected planet (dot symbols) from Gilli et al. (2006) are shown on the $\left([\mathrm{Fe} / \mathrm{H}], V_{\text {rot }}\right)$ distribution of Fig. 1. The kinematic parameters are from the GCS catalogue. We emphasize that all stars known to harbour planets at $[\mathrm{Fe} / \mathrm{H}]<-0.20 \mathrm{dex}$ and with available estimates of the $[\alpha / \mathrm{Fe}]$ ratio (therefore excluding $M$ dwarfs) are included in the sample, and are shown as large star symbols in Fig. 1a. A planet orbiting the metal-poor star HD 171028 has recently been reported (Santos et al. 2007). HD 171028 is not an Hipparcos star, and its distance is uncertain (Nordström et al. report 90 pc, while Santos et al. give 43 pc), and so are its velocities. However, the $V$ component $\left(2 \mathrm{~km} \mathrm{~s}^{-1}\right.$ in the GCS catalogue) combined with its metallicity $([\mathrm{Fe} / \mathrm{H}]=$ -0.49 dex $)$ and $\alpha$ element content $([\alpha / \mathrm{Fe}]=0.05 \mathrm{dex})$, confirms that HD 171028 is a metal-poor thin disc star. Although the overall statistics is small at $[\mathrm{Fe} / \mathrm{H}]<-0.20 \mathrm{dex}$, it illustrates that 


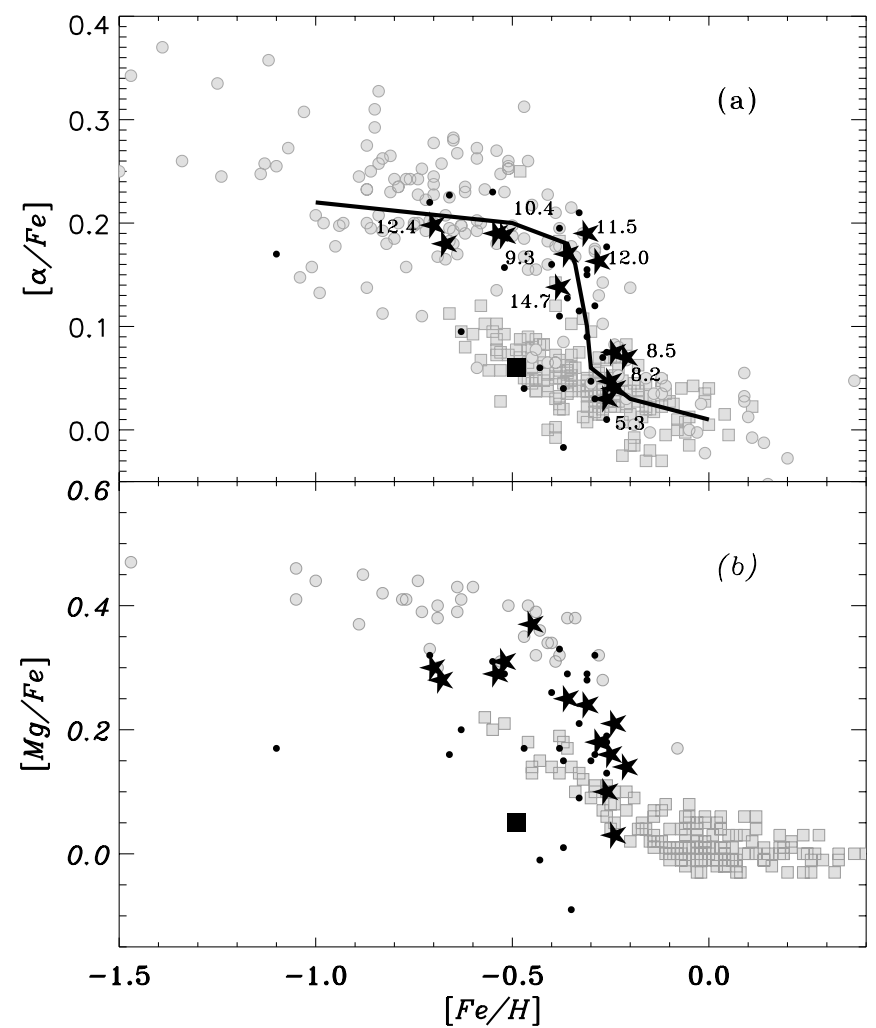

Fig. 2. $[\alpha / \mathrm{Fe}]$ a) and $[\mathrm{Mg} / \mathrm{Fe}]$ b) as a function of $[\mathrm{Fe} / \mathrm{H}]$ for stars with planets considered in this paper (star symbols). Stars with no detected planet are represented by dots. The square symbol represents the position of HD 171028. Grey symbols (squares or circles for kinematically defined thin or thick discs respectively as defined by each study) are field stars from Reddy et al. (2003, 2006) on plot a), and Fuhrmann $(1998,1999,2004)$ on plot b). The thick line is only indicative and illustrates the evolutionary sequence from the thick disc to the thin disc.

stars with planets are found essentially in the group lagging the LSR, either in the "thick disc" population or intermediate between the thin disc and the thick disc, with only one object in the group of metal-poor thin disc stars.

\subsection{2. $\alpha$-element ratio}

Stars of the thin and thick discs show distinct properties in their level of $\alpha$ enrichment as a function of metallicity. This is illustrated in studies of Fuhrmann (1998) and Reddy et al. (2003, 2006) (among others), which samples are displayed in Fig. 2. The hiatus between the metal-poorest stars of the thin disc and the thick disc is apparent in these two plots (metal-poor thin disc stars at $([\mathrm{Fe} / \mathrm{H}], \alpha / \mathrm{Fe}])=(-0.6,0.1)$ dex metal-rich thick disc at $(-0.2,0.15)$ dex). It is however well explained (Haywood, 2007) because metal-poor thin disc stars (located in Fig. 2a at $[\mathrm{Fe} / \mathrm{H}]<-0.3 \mathrm{dex}$ and $[\alpha / \mathrm{Fe}]<0.1 \mathrm{dex})$ are identified as the stars with $V_{\text {rot }}>-5 \mathrm{~km} \mathrm{~s}^{-1}$ in Fig. 1a. In other words, these objects have probably formed outside the solar circle, and probably are not relevant tracers of the chemical evolution at the solar radius. If we discard these stars in Fig. 2a, the other objects form a continuous sequence from the thick disc to the thin disc, materialised by the continuous curve.

The two samples of stars with and without planets (limited to $[\mathrm{Fe} / \mathrm{H}]<-0.2$ dex $)$ are plotted in Figs. 2a, b. The dichotomy between the two is apparent. To the notable exception of HD 171028, stars with planets have a tendancy to avoid the area of metal-poor thin disc stars, and are positioned on the
Table 1. Stars with planets considered in this study.

\begin{tabular}{|c|c|c|c|c|c|}
\hline$\overline{\mathrm{Id}}$ & {$[\mathrm{Fe} / \mathrm{H}]$} & $T_{\text {eff }}$ & $\overline{[\alpha / \mathrm{Fe}]}$ & Age & $\bar{V}$ \\
\hline HIP & dex & $K$ & $\operatorname{dex}$ & Gyr & $\mathrm{km}^{-1}$ \\
\hline 3479 & -0.24 & 5626 & 0.04 & & -4 \\
\hline 3497 & -0.33 & 5636 & 0.19 & $11.5(6.5,16.1)$ & -109 \\
\hline 5054 & -0.52 & 5835 & 0.188 & $10.4(5.6,14.8)$ & -67 \\
\hline 10138 & -0.24 & 5163 & 0.075 & & -75 \\
\hline 26381 & -0.38 & 5546 & 0.138 & $14.7(8.2,20.0)$ & -24 \\
\hline 31688 & -0.54 & 4554 & 0.19 & $9.3(7.4,11.2)$ & \\
\hline 58952 & -0.28 & 4773 & 0.163 & & -8 \\
\hline 62534 & -0.36 & 5494 & 0.17 & $12.0(4.319 .5)$ & -86 \\
\hline 64426 & -0.70 & 5884 & 0.198 & $12.4(9.5,15.0)$ & -69 \\
\hline 64459 & -0.25 & 5886 & 0.047 & $8.2(6.3,10.0)$ & -86 \\
\hline 78459 & -0.21 & 5853 & 0.07 & $8.5(5.8,10.7)$ & -36 \\
\hline 83949 & -0.67 & 5868 & 0.18 & & -53 \\
\hline 98714 & -0.26 & 5327 & 0.03 & $5.3(4.5,6.3)$ & -48 \\
\hline HD 171028 & -0.49 & 5663 & 0.05 & & 2 \\
\hline
\end{tabular}

Notes: Atmospheric parameters from Gilli et al. (2006) or Soubiran \& Girard (2005) for HIP 3497 and HIP 83949. $V$ velocities from Nordström et al. (2004). Ages are derived as described in the text, except for HIP 31688, which age comes from da Silva et al. (2006). The ages of HIP 58952 (giant), HIP 3479 and HIP 10138 could not be determined. Parameters of HD 171028 from Santos et al. (2007) are given.

intermediate path between the thin and thick discs (between -0.3 and $-0.2 \mathrm{dex}$ ), then in the thick disc regime (at $[\mathrm{Fe} / \mathrm{H}]<$ $-0.3 \mathrm{dex}$ and $[\alpha / \mathrm{Fe}]>0.15 \mathrm{dex}$ or $[\mathrm{Mg} / \mathrm{Fe}]>0.2 \mathrm{dex})$. Note that among stars with planets which have the lowest amount of $\alpha$-elements, HIP 98714 is unambiguously classified as a transition or old thin disc stars (not as a metal-poor thin disc with outer-disc origin), according to its lag on the $V$ component $\left(-48 \mathrm{~km} \mathrm{~s}^{-1}\right)$, the same for HIP 64459 (at $-86 \mathrm{~km} \mathrm{~s}^{-1}$ ), while HIP 3479 is more intermediate $([\mathrm{Fe} / \mathrm{H}]=-0.24$ and $V_{\text {lag }}=-4 \mathrm{~km} \mathrm{~s}^{-1}$ ) between the two groups.

\subsubsection{The age of metal-poor stars with planets}

Stars with planets in the metallicity range of interest here and which could be dated are shown in Fig. 2a with their ages (see also Table 1). Stars were dated using the procedure described by Jørgensen \& Lindegren (2005) with the isochrone set of Demarque et al. (2004), atmospheric parameters from Table 1 and absolute magnitudes from Hipparcos parallaxes. HD 171028 could not be dated to useful precision (due to the uncertainty of its parallax). The age distribution of stars with planets does not contradict the general scheme just presented. If we adopt the general scenario described in studies of solar neighbourhood stars (Bernkopf et al. 2001; Haywood 2006, 2007) the thin disc has emerged from a transition phase with the thick disc at an age of about 8 Gyr. It is unknown how long this transition phase has lasted, and ages of thick disc stars are uncertain (9-13 Gyr). However, Fig. 2a illustrates that stars with planets roughly follow this sequence, from stars of the old thin disc at $[\mathrm{Fe} / \mathrm{H}] \approx-0.2$ dex and ages of about $8 \mathrm{Gyr}$ to stars in the thick disc regime, enriched in $\alpha$-elements and older by a few Gyr.

\section{Discussion}

We find there is only 1 star with planets in the metal-poor thin disc population, namely those stars more metal-poor than approximately -0.2 to -0.3 dex, having low level of $\alpha$-element enrichment and mostly preceding the LSR. Note that the approximate metallicity interval $[-0.3,-0.2]$ dex may contain a mixture 
of interlopers from the outer disc and genuine solar galactocentric stars. Are the above results significant? The total number of stars in the two groups (metal-poor thin disc and thick disc or intermediate stars, as separated by a limiting lag of $-5 \mathrm{~km} \mathrm{~s}^{-1}$ ) in $V$, at a metallicity smaller than $-0.20 \mathrm{dex}^{1}$, are respectively 9 and 29 stars, with 1 and 13 stars in each group harbouring planets. Note that the number of stars with no detected planet in the group of metal-poor thin disc objects cannot be taken as indicative of the number of surveyed stars in this group, because no exhaustive list has been published up to now. The consequence is that the significance of our result must be evaluated on the number of planet found in each group.

There are, in principle, two different ways to proceed. If we knew the total number of stars surveyed in each of the two groups (therefore, the total number of stars with no detected planet), we could estimate whether the difference of detected planets (13 and 1) is significant. However, these figures are not publicly available. Moreover, we would need information on the $\alpha$-element content of the stars, (since it is the best criterion to discriminate between the two groups), but this is measured only on a restricted number of objects.

Another way to proceed is to assume that no selection criterion that could favour the inclusion of metal-poor thin disc or thick disc stars have been adopted in exoplanet surveys. Then we may estimate the expected relative number of stars in each of the two groups, and consecutively infer that, if the probability of finding an exoplanet depends only (or mainly) on metallicity, the relative proportion of detected exoplanet in the two groups should follow the same ratio. The relative normalisation of the two groups of stars can be obtained from a distance-limited sample. The data set of Fuhrmann (2004) is designed to be complete for FGK dwarfs up to $25 \mathrm{pc}$. It contains by now about 250 stars (out of approximately 300 objects when completed) and according to the author it is representative of the final sample. 8 stars in the sample of Fuhrmann (2004) clearly belong to the thick disc and have corresponding level of $[\mathrm{Mg} / \mathrm{Fe}](>0.3 \mathrm{dex})$ and one is intermediate. 12 stars belong to the thin disc metal-poor tail and have $[\mathrm{Fe} / \mathrm{H}]<-0.3 \mathrm{dex}$ (which, given the Poissonian uncertainties, can be considered as equivalent). We assume conservatively an equal probability of finding a star in the metal-poor thin disc or in the thick disc. It implies that we should expect the same number of detected planets in the two groups. What is then the probability that, if the proportion of stars with planets is about the same in the two groups, 13 out of 14 found so far in this metallicity range belong to one group only? The binomial probability function gives a probability of $8 \times 10^{-4}$ that this could have occured by chance. In other words, at a given metallicity, stars are less likely to harbour a giant planet if they originate from the outer Galactic disc than a star born at solar galactocentric radius. This result must be considered together with the fact that metal-rich stars, which are the hosts of most of the detected planets, have kinematic properties suggesting an origin in the inner disc. In other words, stars originating in the outer disc have the lowest rate of detected planets, while stars coming from the inner disc show the highest rate, stars at the solar circle being intermediate between these two extremes.

These facts suggest that, being apparently correlated to metallicity, the presence of giant planets might be primarily dependent of some specific property of the ISM at the original galactocentric distance of the star. Although theoretical modeling suggests that metallicity is a key parameter (cf. references in the Introduction), it is possibly not the single parameter, and perhaps not even a parameter related to the presence of a planet, since otherwise we would expect similar proportions in the two groups studied here. On the other hand, a parameter more strongly correlated with galactocentric distance than metallicity must play a role. Giving a possible candidate would be hasardous at this stage, but inspecting the radial variation of some specific ingredients (dust?) of the ISM would be worthwhile.

It must be noted that the apparent correlation between metallicity and the detection of planets is a natural consequence of these results. Since the mean metallicity of field stars increases towards the galactic center, planets forming preferentially on stars in the inner disc will form on metal-rich stars. Radial mixing is then responsible for bringing these stars to the solar circle. The dependency with some other specific property related to the radial distance to the galactic center (other than metallicity) could also explain the peculiarity found at lower metallicity, which cannot be accounted for by the simple dependence with metallicity. If these inferences are correct, we can also evaluate the proportion of stars with exo-planets that truly originate from the solar cicle. The mean metallicity of the Galactic disc in the solar neighbourhood is about $[\mathrm{Fe} / \mathrm{H}]=0.0$ dex (Haywood 2001), while the terminal metallicity of stars at solar galactocentric radius (the upper most metallicity reached by stars born at solar radius), is about 0.15 dex (Haywood 2006). Only half of the stars with detected planets have a metallicity below this limit, and can be considered as truly endemic to the solar circle.

Acknowledgements. I would like to thank Frédéric Arenou and ALM for their questions and suggestions which much improved this article. Thanks are also given to the referee for his helpful comments.

\section{References}

Barbieri, M., \& Gratton, R. G. 2002, A\&A, 384, 879

Bensby, T., Feltzing, S., Lundström, I., \& Ilyin, I. 2005, A\&A, 433, 185

Bernkopf, J., Fidler, A., \& Fuhrmann, K. 2001, ASPC, 245, 207

da Silva, L., Girardi, L., Pasquini, L., et al. 2006, A\&A, 458, 609

Demarque, P., Woo, J.-H., Kim, Y.-C., \& Yi, S. K. 2004, ApJS, 155, 667

Ecuvillon, A., Israelian, G., Pont, F., Santos, N., \& Mayor, M. 2007, A\&A, 461, 171

Famaey, B., Pont, F., Luri, X., et al. 2007, A\&A, 461, 957

Fuhrmann, K. 1998, A\&A, 338, 161

Fuhrmann, K. 1999, Ap\&SS, 265, 265

Fuhrmann, K. 2004, AN, 325, 3

Gilli, G., Israelian, G., Ecuvillon, A., Santos, N., \& Mayor, M. 2006, A\&A, 449, 723

Haywood, M. 2001, MNRAS, 325, 1365

Haywood, M. 2006, MNRAS, 371, 1760

Haywood, M. 2007, A giant step: from milli to microarcsecond astrometry, IAU Symp., 248

Ida, S., \& Lin, D. N. C. 2005, PThPS, 158, 68

Jørgensen, B. R., \& Lindegren, L. 2005, A\&A, 436, 127

Laughlin, G. 2000, ApJ, 545, 1064

Mordasini, C., Alibert, Y., Benz, W., \& Naef, D. 2007, [arXiv: 0710. 5667]

Nordström, B., Mayor, M., Andersen, J., et al. 2004, A\&A, 418, 989

Reddy, B., Tomkin, J., Lambert, D., \& Allende Prieto, C. 2003, MNRAS, 340, 304

Reddy, B., Lambert, D., \& Allende Prieto, C. 2006, MNRAS, 367, 1329

Robinson, S. E., Laughlin, G., Bodenheimer, P., \& Fischer, D. 2006, ApJ, 643, 484

Santos, N. C., Israelian, G., \& Mayor, M. 2000, A\&A, 363, 228

Santos, N. C., Mayor, M., Bouchy, F., et al. 2007, A\&A, 474, 647

Sellwood, J. A., \& Binney, J. J. 2002, MNRAS, 336, 785

Udry, S., \& Santos, N. C. 2007, ARA\&A, 45, 397

Valenti, J. A., \& Fischer, D. A. 2005, ApJS, 159, 141

\footnotetext{
1 The limiting metallicity adopted in Sect. 2.1 was -0.35 dex. We now move to -0.20 dex because $\alpha$-element content allow a correct separation of the two groups of stars up to this metallicity.
} 